\title{
The Regulation of Dietary Iron Bioavailability by Vitamin C: A Systematic Review and Meta-Analysis
}

\author{
A. Heffernan, C. Evans, M. Holmes and J.B. Moore \\ School of Food Science and Nutrition, University of Leeds, Leeds, West Yorkshire, LS2 9JT, UK
}

Iron deficiency is the most common nutritional deficiency worldwide, in part due to the inherent low bioavailability of dietary iron ${ }^{(1)}$. Although dietary components such as vitamin $\mathrm{C}$ have been demonstrated to influence iron bioavailability, with the discovery of hepicidin as the hormone central regulator of iron homeostasis ${ }^{(2)}$, their significance has been questioned. Specifically, the Scientific Advisory Committee in the UK has stated that only the consumption of a healthy balanced diet is required for adequate iron status and the role of dietary enhancers is minimal ${ }^{(3)}$. Therefore, the aims of this systematic review and meta-analysis were to determine the effect of vitamin $\mathrm{C}$ on nonheme iron absorption and the effects of long term supplementation on iron status.

The review was conducted by independent reviewers in accordance with PRISMA guidelines and is registered at Prospero (CRD42014010453). Medline, Scopus and Cochrane databases were searched from inception through to December $31^{\text {st }} 2016$, with a basic search strategy of "(iron OR ferritin OR haemoglobin) AND (ascorbic acid OR ascorbate OR vitamin C)". Short-term ( $<4$ weeks) and long-term trials ( $>4$ weeks) reporting measures of iron absorption from isotopically labelled iron meals with and without vitamin C or changes to biomarkers of iron status with vitamin C supplementation in healthy adults were included. Meta-analyses were executed in Review Manager 5 software (version 5.3).

From a total of 4830 identified records, 26 studies ( 20 short-term, 6 long-term) were included in qualitative synthesis and 22 were included in the meta-analysis ( 3 excluded as reported arithmetic not geometric means, 1 excluded for not reporting variance). All of the short-term studies were a 16-day crossover design, where the same iron test meals were labelled with different isotopes and consumed on consecutive mornings with and without ascorbic acid; with washout in between. The combined pooled effects showed a highly significant increase in \% iron absorption when ascorbic acid was added to test meals and the $1^{2}$ statistic quantifying the level of heterogeneity, indicated a very low level of inconsistency (MD: 5.87; $95 \%$ CI 4.43, 7.31; $\mathrm{P}=0.00001 ; \mathrm{n}=315$ total study participants; $1^{2}=14 \%$ ).

The effect of long-term ascorbic acid supplementation on haemoglobin status was assessed in 5 studies, arranged in descending order of haemoglobin $(\mathrm{Hb})$ level $(\mathrm{g} / \mathrm{L})$ which ranged from $146 \mathrm{~g} / \mathrm{L}-101 \mathrm{~g} / \mathrm{L}$ (Fig. 1). These data show ascorbic acid supplementation increased haemoglobin value levels from baseline to follow-up significantly, $(\mathrm{MD}: 8 \cdot 26 ; 95 \% \mathrm{CI} 3 \cdot 00,13 \cdot 53 ; \mathrm{P}=0 \cdot 002 ; \mathrm{n}=118$ total study participants; $1^{2}=58 \%$ ), with a moderate level of inconsistency between studies.

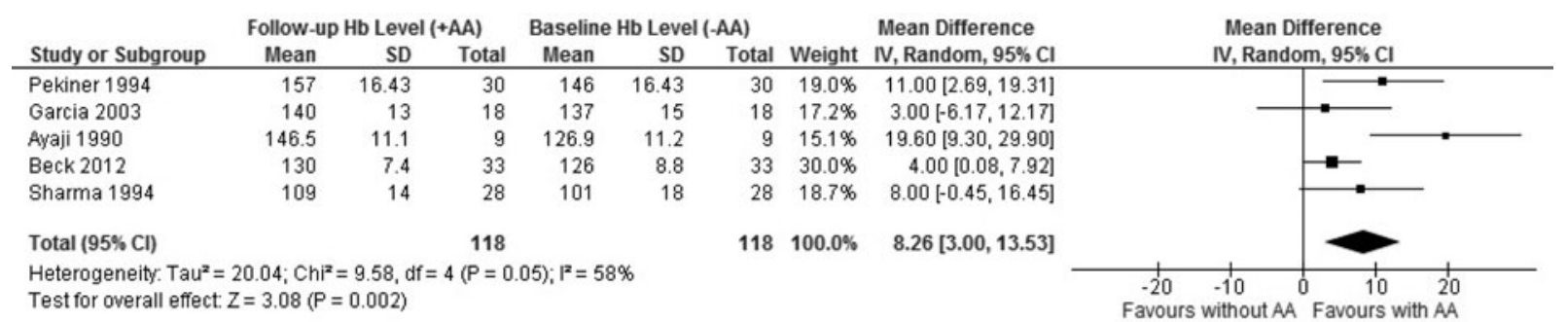

Fig. 1. Forest plot showing the effect of ascorbic acid (AA) on haemoglobin ( $\mathrm{Hb}, \mathrm{g} / \mathrm{L})$ status.

We conclude, ascorbic acid enhances the absorption of nonheme iron and can increase biomarkers of iron status overtime. Increasing vitamin $\mathrm{C}$ intake from food or supplementation remains a practical measure to reduce the risk of iron deficiency and to moderate the incidence of iron deficiency anaemia.

1. Camaschella C (2015) NEJM 372, 1832-1843.

2. Ganz T. (2011) Blood 117(17): 4425-4433.

3. Scientific Advisory Committee on Nutrition (2010) Iron and Health. London: TSO. 